\title{
The Potential Role of Changes in the Glucose and Lipid Metabolic Pathways in Gastrointestinal Cancer Progression: Strategy in Cancer Therapy
}

\author{
Gordon A. Ferns ${ }^{\mathrm{a}}$ Milad Shahini Shams Abadi ${ }^{\mathrm{b}, \mathrm{c}}$ Ahmad Raeisi $^{\mathrm{d}}$ \\ Mohammad-Hassan Arjmand ${ }^{c, e}$
}

${ }^{a}$ Division of Medical Education, Brighton \& Sussex Medical School, Brighton, UK; ${ }^{b}$ Department of Microbiology and Immunology, Cellular and Molecular Research Center, Basic Health Sciences Institute, Shahrekord University of Medical Sciences, Shahrekord, Iran; 'C Cancer Research Center, Shahrekord University of Medical Sciences, Shahrekord, Iran; ${ }^{d}$ Clinical Research Development Unit, Hajar Hospital, Shahrekord University of Medical Sciences, Shahrekord, Iran; eMedical Plants Research Center, Basic Health Sciences Institute, Shahrekord University of Medical Sciences, Shahrekord, Iran

\section{Keywords}

Cancer metabolism · Gastrointestinal tumors · Glucose metabolism · Lipid metabolism

\section{Abstract}

Background: Changes in cell metabolism are a well-known feature of some cancers, and this may be involved in the etiology of tumor formation and progression, as well as tumor heterogeneity. These changes may affect fatty acid metabolism and glycolysis and are required to provide the increase in energy necessary for the high rate of proliferation of cancer cells. Gastrointestinal cancers remain a difficult-to-treat cancer, particularly as they are usually diagnosed at a late stage of disease and are associated with poor outcomes. Summary: Recently, the changes in the metabolic pathways, including the expression of the rate-limiting enzymes involved, have been considered to be a potential target for therapy for gastrointestinal tumors. Key Message: A combination of routine chemotherapy drugs with metabolic inhibitors may improve the effectiveness of treatment.

(C) 2021 The Author(s).

Published by S. Karger AG, Basel

\section{Introduction}

Cancer cells are irregular cells with unlimited proliferation, transformation, and migration that lead to more energy requirements by unusual metabolic pathways that are more active than normal cells. The interaction between metabolic pathways and solid tumor growth has been investigated in several studies $[1,2]$. The cellular composition of solid tumors is often heterogeneous and comprises different cell types within the tumor microenvironment [3]. Hence, solid tumors display different metabolic landscapes due to some intrinsic factors and also signaling to rise by the microenvironment [4]. Importantly, different metabolic pathways in tumor cells and stroma tissues around tumor cells support the development of a metabolic symbiosis between tumor cells and the tumor microenvironment [5]. Gastrointestinal (GI) malignancies are an important global public health problem. Patients with GI cancers are often diagnosed at a late stage and consequently have a poor prognosis outcome [6]. The mechanisms of tumor progression in GI tumors are a major focus of research. Regulatory changes of the metabolic network in tumor cells may help to explain the
Correspondence to:

Mohammad-Hassan Arjmand, arjmandmh1@gmail.com 
mechanisms behind the tumor therapeutic failures and determine clinical outcomes. In this review, we summarize the metabolic alterations and their effects on a different aspect of cancer biology in GI malignancies.

\section{Tumor Cell Energy and Metabolism}

A common and important feature of malignant tumor cells is the deregulation of some metabolic pathways that are involved in energy production [7]. High energy demand and synthesis of macromolecules are due to the high proliferative rates of cancer cells [8]. Therefore, tumor cells need to reprogram their metabolic pathways to enable energy production and sustain their growth rate $[8,9]$.

\section{Glucose Metabolism}

The Warburg effect is the increased conversion of glucose to lactate in relation to low oxidative profile due to aerobic glycolysis in tumor cells [10]. The Warburg effect is a metabolic feature in most tumor cells and gives several benefits to the tumor cells that include increased survival ability during changes in tissue oxygen levels; moreover, some mediators produced in glycolysis are used in other pathways like nucleotide and lipid biosynthesis and acidify the tumor microenvironment that affects the activity of other cells like immune cells $[11,12]$. Moreover, hypoxia is a common feature of malignant tumors and contributes to cancer progression and drug resistance [13]. Hypoxic conditions activate the hypoxia-inducible factor $1 \alpha$ (HIF-1 $\alpha)$ to stimulate metabolic reprogramming and induce glycolytic activity $[12,13]$. HIF-1 and c-Myc as transcriptional factors target most glycolic enzyme genes such as hexokinases (HK), pyruvate kinase (PK), and pyruvate dehydrogenase kinase (PDK). Several studies have shown that the significance of HIF-1 $\alpha$ - and c-Myc-positive expression is associated with poor prognostic outcomes in patients with GI cancers such as pancreatic adenocarcinoma (PADC) [14] and gastric cancer (GC) [15].

HK, the first enzyme in the glycolytic pathway, phosphorylates glucose to glucose 6 phosphates and retains glucose in cells. $\mathrm{HK}$ has 4 isoenzymes $\left(\mathrm{HK}_{1-4}\right) . \mathrm{HK}_{2}$ is a major isoform and unregulated in many tumor types compared with normal tissue [16]. Several studies have demonstrated that high expression of $\mathrm{HK}_{2}$ is associated with poor prognosis in different GI cancers such as GC and $\mathrm{HCC}[17,18]$. Increased $\mathrm{HK}_{2}$ expression was reported to be associated with the advanced stage of $\operatorname{HCC}[18,19]$.
A previous study indicated that the use of 3-bromopyruvate inhibited $\mathrm{HK}_{2}$ expression and reduced the proliferation of colorectal cancer (CRC) cell lines when combined with daunorubicin [20].

Pyruvate, a final product of the glycolytic pathway, is generated from phosphoenolpyruvate by PK. Four isoforms of PK have been identified, but the $\mathrm{M}_{2}$ isoform of $\mathrm{PK}$ is essential for aerobic glycolysis and expressed in cancer cells, adult stem cells, and embryonic cells [21, 22]. $\mathrm{PKM}_{2}$ as a kinase leads to phosphorylation of histone $\mathrm{H} 3$ in the nucleus and contributes to tumor genesis [23]. Also, PKM has a role in the biosynthesis of nucleic acid tumor progression [24]. $\mathrm{PKM}_{2}$ overexpression is related to poor outcomes in ESCC and GC $[25,26]$. Also, upregulation of $\mathrm{PKM}_{2}$ reduces the levels of electron transport chain complex proteins and so contributes to glycolysis and can damage the normal function of mitochondria [27]. $\mathrm{PKM}_{2}$ is a potential target molecule for cancer treatment and suggests that a combination of PKM2 inhibitors with other targeted therapies like gefitinib can increase the potential of cancer treatment. Shikonin, a natural naphthoquinone, significantly inhibits the glycolytic rate through PK inhibition. Many studies have demonstrated that shikonin can decrease multiple types of cancers, including esophageal and hepatocellular cancer $[28,29]$.

Several investigations have reported an association between PDK positivity and prognosis in GI cancer. PDK inhibits pyruvate dehydrogenase activity by phosphorylation and increases the generation of pyruvate to lactic acid. PDK has 4 isoforms, and their expression is organ specific. Overexpression of $\mathrm{PDK}_{1}$ was shown in GC and related to poor survival [30]. Moreover, an increase in the expression of $\mathrm{PDK}_{3}$ was detected in CRC [31]. Dichloroacetic acid, a PDK-1 inhibitor, decreased lactate generation and promoted responsiveness to 5-FU in CRC cell lines [32]. Treatment of HCC cell lines with dichloroacetic acid reduces cell proliferation and also decreases sorafenib resistance [33].

Phosphofructo-1-kinase (PFK1) is a key regulatory enzyme in the glycolytic pathway that converts glucose 6 phosphates into glucose 1-6 bisphosphate and adenosine diphosphate. Liver type (PFK-L), muscle type (PFK-M), and platelet type (PFK-P) are 3 important isoforms of PFK in humans $[34,35]$. PFK1 activity is induced in response to proliferation signaling that increases glycolysis in cancer cells [35]. PFK-L and PFK-P are the main isoenzymes that are elevated in tumor cells $[36,37]$. In addition, PFK1 activity is increased by HIF-1 $\alpha$ in malignancies [38]. Because of the increased dependence of cancer cells on the glycolytic 
Fig. 1. Diagram showing the expression of glycolytic enzymes and GluTs in tumor and normal cells and also small-molecule inhibitors in the Warburg effect for cancer therapy. In normal cells, pyruvate is usually transported to the mitochondria for TCA and oxidative phosphorylation, but in tumor cells, Warburg effect increases lactate by converting most of pyruvate to lactate. TCA, tricarboxylic acid.

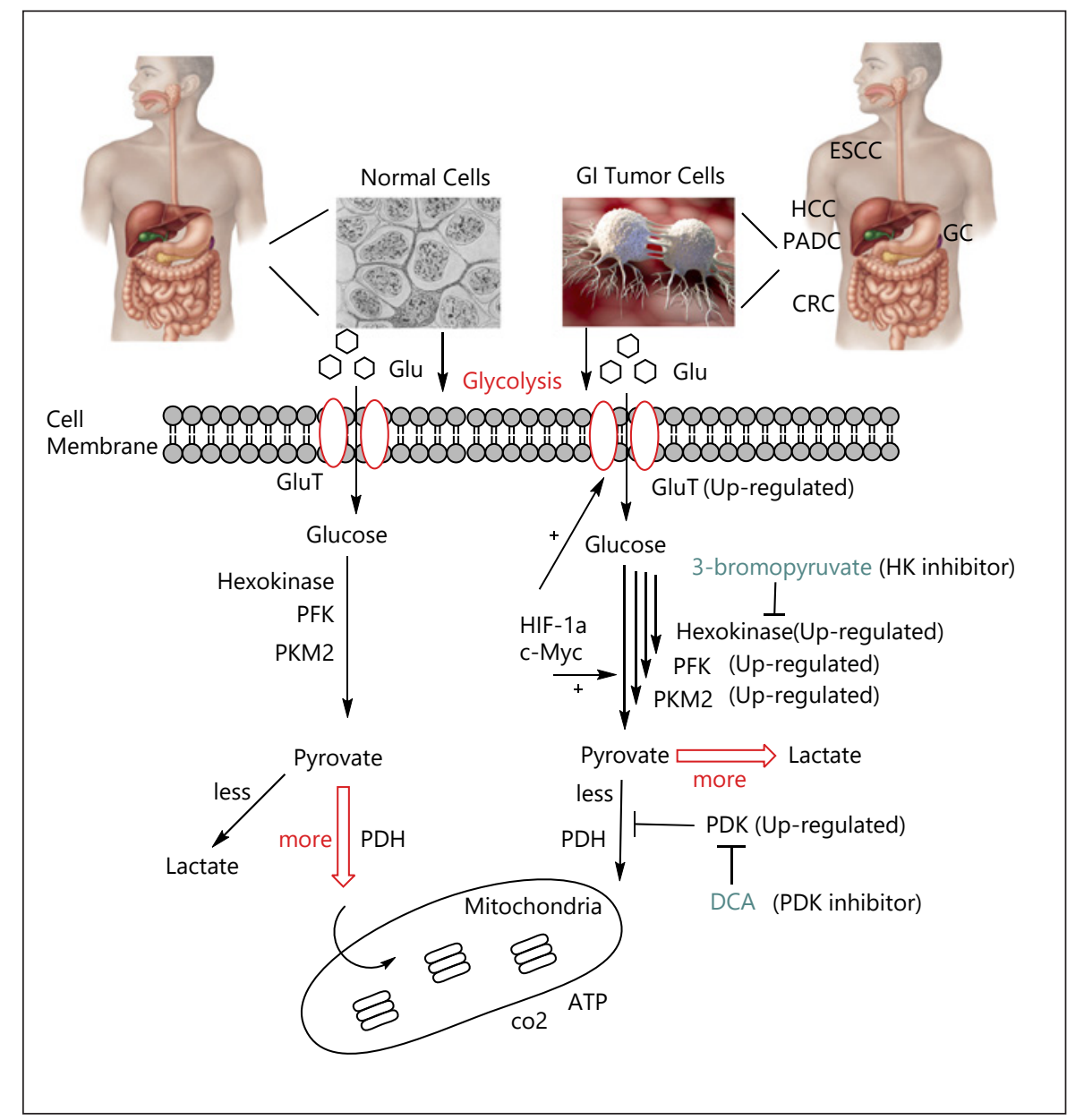

pathway, PFK can be an effective treatment option of cancer. Zhu et al. [39] have shown that 3-(3-pyridinyl)-1-(4pyridinyl)-2-propen-1-one as a PFK inhibitor induces apoptosis and inhibits invasion of GC cells.

Glucose transporters (Glut) are membrane proteins responsible for the uptake of glucose into the cells across the plasma membrane and are different for a specific tissue. Glut1-4 isoforms are the most common studies in solid neoplasms. Different studies indicated that Glut1 is overexpressed in CRC and is associated with poor survival in patients $[40,41]$. In GC, the analyzed samples showed that Glut1 and Glut3 are increased, and the expression of Glut3 was associated with higher stage and poor outcomes [42]. Other studies have demonstrated that Glut1 is upregulated in pancreatic cancer and esophageal adenocarcinoma, and the expression level was associated with clinicopathological features and prognosis $[43,44]$. Glut 1 positivity was related to rectal cancer tumor grade and maybe a suitable prognostic biomarker for evaluation to chemoradiotherapy. Furthermore, c-Myc and HIF-1 $\alpha$ increase the expression of Glut1 and 3 to increase glucose uptake in cancer cells and induce tumor cell growth $[45,46]$.

Different studies have shown that glycolytic enzyme genes like HK2, PKM2, PDK1, and Glut are targeted by transcriptional factors such as HIF-1 $\alpha$ and C-Myc, so more investigations about the molecular mechanisms of these genes may provide insights about the tumor biology and treatment. In Figure 1, we show a schematic picture of the glycolytic pathway and involved molecules in the Warburg effect in the tumor cells. Also, we summarize the most important regulatory enzymes and molecules in the glycolytic pathway and their inhibitors against GI malignancies in Table 1.

\section{Lipid Metabolism}

Lipids, a highly different class of biological molecules, are necessary components in cell function and 
Table 1. Summary of regulatory enzymes related to the glucose and lipid metabolic pathway and some inhibitors against GI malignancies

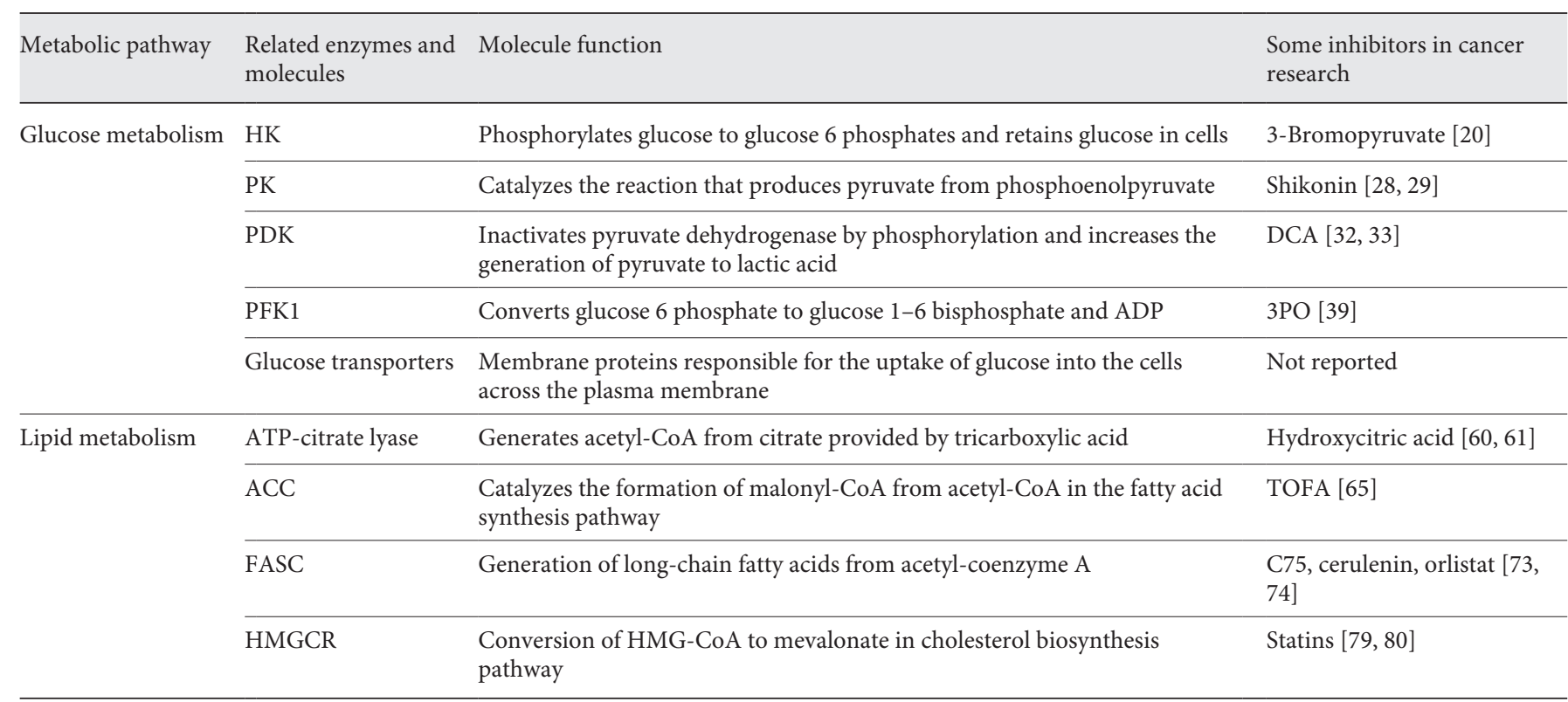

HK, hexokinases; PK, pyruvate kinase; PDK, pyruvate dehydrogenase kinase; DCA, dichloroacetic acid; PFK1, phosphofructo-1-kinase; 3PO, 3-(3-pyridinyl)-1-(4-pyridinyl)-2-propen-1-one; ACC, acetyl-CoA carboxylase; TOFA, 5-(tetradecyloxy)-2-furoic acid; FASC, fatty acid synthase complex; HMGCR, hydroxy-3-methylglutaryl-CoA reductase.

have different biological roles such as a structural role in a biological membrane, metabolism, and energy storage and also as signaling molecules to regulate many cellular activities. The regulation of lipid metabolisms such as lipid biosynthesis, lipid uptake, and lipid hydrolysis is needed for cellular hemostasis [47]. During tumor progression, there is a change in the tumor microenvironment, around the tumor cells, in which there is a change in lipid metabolic pathways in taking energy that contributes to the rapid cell proliferation and increases survival and migration of cancer cells. The role of lipid metabolism variations in tumor progression has been clearly shown [48].

Obesity is a risk factor for several cancer types. Obesity is defined as excess visceral fat, and obesity can be considered as a chronic disorder. Excess visceral depots of adipose tissue in obesity may cause alterations in the lipid metabolism in the tumor cell microenvironment contributing to tumor cell proliferation and migration $[49,50]$. The release of fatty acids (FAs) and proinflammatory cytokines around the tumor cells may be caused by adipocytes, and this may support tumor cell progression [49]. Moreover, there is excessive production of arachidonic acid by adipose tissues in obesity, and this FA is a key precursor lipid for the production of proinflamma- tory molecules like leukotrienes, and prostaglandins promote tumor cell development $[51,52]$. Studies have demonstrated that obesity is associated with GI malignancies such as CRC [53], liver [54], and PADC [55].

De novo FA biosynthesis occurs predominantly in the liver and adipose tissue. ATP-citrate lyase generates acetyl-CoA from citrate provided by the tricarboxylic acid cycle. Production of acetyl-CoA is the essential step of FA biosynthesis. Activation of acetyl-CoA to malonyl-CoA and coupled acetyl and malonyl to the acyl-carrier protein are needed to produce palmitic acid and other kinds of FAs. De novo FA synthesis has been associated with tumor cell proliferation and metastasis in different cancers [56]. It has been demonstrated that ATP-citrate lyase is needed for cell transformation in vitro and for tumor genesis in vivo [57]. ATP-citrate lyase is a bridge between the glycolytic cascades and starting lipid metabolism. Likewise, connections between glucose metabolisms with lipogenesis through ATP-citrate lyase enable tumor cells to grow, and it could offer a novel therapeutic target. Qian et al. [58] have shown that high ATP-citrate lyase expression was associated with advanced stages of cancer and lymph node metastasis in patients with GC, and patients with low expression of ATP-citrate lyase had better survival time. Furthermore, Zhou et al. [59] have shown that 
the expression of ATP-citrate lyase was upregulated in CRC in comparison with normal tissues, and it was correlated with chemoresistance in patients. Also, they found that targeting ATP-citrate lyase can improve the therapeutic effects and increase the survival outcomes. Several studies have shown that hydroxycitric acid is able to suppress CRC, suppressed growth, migration, and invasion by ATP-citrate lyase inhibition $[60,61]$. One of the other important enzymes in FA biosynthesis is acetyl-CoA carboxylase (ACC). ACC is a biotin-dependent enzyme that catalyzes the formation of malonyl-CoA in the FA synthesis pathway [62]. Fang et al. [63] have reported a significant relationship between high expressions of ACC with clinicopathological parameters in GC and have the potential to introduce as a prognostic factor. In another investigation, Calvisi et al. [64] have detected the upregulation of active ACC in HCC. Moreover, several studies have described that hypoxia conditions around the tumor cells induce the expression of enzymes related to FA biosynthesis like ACC. These findings suggest that ACC can be a molecular target for GI cancer therapy. 5-(Tetradecyloxy)-2-furoic acid as an ACC inhibitor induces apoptosis and suppression of human pancreatic cancer cells [65].

Fatty acid synthase complex (FASC) is a crucial enzyme in the lipogenic pathway and the generation of longchain FAs from acetyl-coenzyme A (CoA) and malonylCoA [66]. In normal cells, FASC is strongly regulated by hormones, diet, and growth factors, not in malignant cells, because rapidly proliferating FAs can be synthesized to provide lipids for energy generation and membrane formation [66]. Overexpression of FASC is observed in many cancers associated with the GI system: ESCC, GC, HCC, CRC, and PADC [67-69]. Different studies demonstrated that elevated expression of FASC has been associated with poor prognosis. Some compounds are identified to inhibit FASC such as cerulenin and C75 which have shown significant anticancer activity [70]. Prizer et al. [71] have shown that $\mathrm{C} 75$ has decreased breast tumors in a xenograft breast cancer model in mice. In another study, Shiragami et al. [72] have found that cerulenin was able to induce apoptosis and cause cytotoxicity in murine CRC cells and in a murine xenograft model; likewise, they have indicated that cerulenin was able to reduce human CRC cell line HCT116 through increased cytotoxicity of cancer cells in vitro. Herein, they have shown that a combination of cerulenin with oxaliplatin has synergistic cytotoxicity effects and can reduce the dosage of oxaliplatin in the treatment of human CRC [73]. Moreover, similar findings have been demonstrated that $\mathrm{C} 75$ decreases the

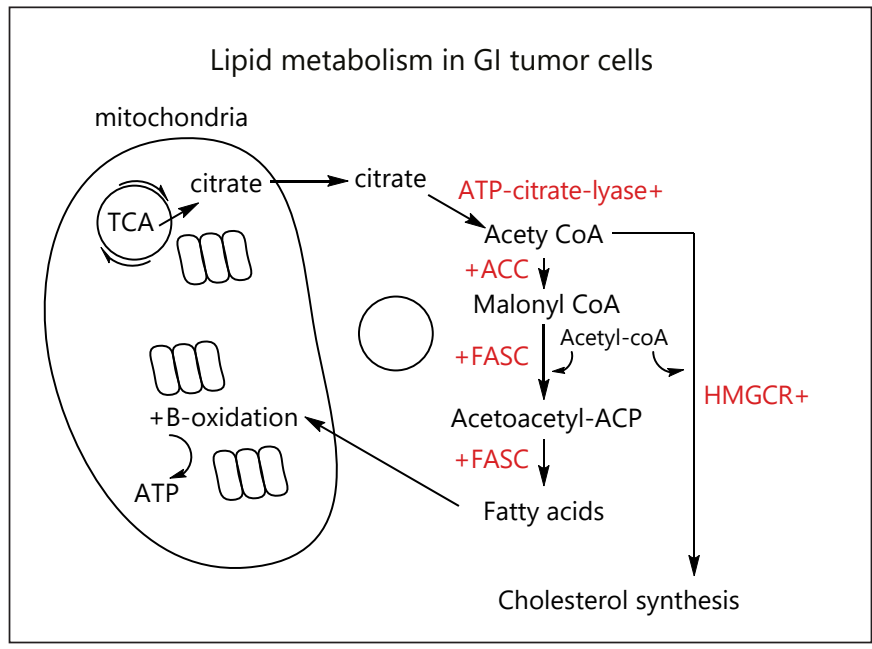

Fig. 2. Main metabolic pathways related to lipid metabolism in GI malignancies. GI, gastrointestinal; ACC, acetyl-CoA carboxylase; FASC, fatty acid synthase complex; HMGCR, hydroxyl methylglutaryl-CoA reductase.

chemosensitivity of GC cells to oxaliplatin by regulating FASN expression [74]. These findings suggest that FASC inhibitors have the potential to be a target for cancer therapy in the future.

Hydroxy-3-methylglutaryl-CoA reductase (HMGCR) is the rate-limiting enzyme in the cholesterol biosynthesis pathway and is responsible for the conversion of HMGCoA to mevalonate [75]. Some studies have demonstrated that the HMGCR is higher in neoplastic tissues in GI cancers [76, 77]. Shi et al. [78] have shown that statins as an HMGCR are able to inhibit ESCC cells in vitro and in vivo. Zhong et al. [79] have found that overexpression of HMGCR stimulated the growth, invasion, and colony formation of ESCC cells while downregulation of HMGCR decreased the tumorigenicity of ESCC cells. Transcription factor $\mathrm{C}-\mathrm{Myc}$ is the main oncogene that induces the expression of HMGCR in tumor cells [78]. These outcomes highlight the potential of the cholesterol pathway as a target for increasing cancer therapy. The targeting of metabolic enzymes related to lipid metabolism is summarized in Table 1.

Although most tumors take up glucose to support their energy and biosynthetic requirements [80], some tumors are dependent on lipid $\beta$-oxidation to take energy. For example, prostate tumors showed to increase uptake of FAs and induce the $\beta$-oxidation enzymes $[81,82]$. $\beta$-Oxidation has also been indicated to provide ATP production and resistance to oxidative stress by producing the substrate to generate NADPH and glutathione to 
reduce oxidative stress [83]. There are few investigations about the relationship between $\beta$-oxidation pathway activation and GI tumor development and metastasis. Toshima et al. [84] have shown HIF-1 $\alpha$ activated in hypoxia condition increases mitochondrial $\beta$-oxidation and cell survival in HCC. More studies are needed to explore the $\beta$-oxidation influences in GI tumor progression. FA biosynthesis and oxidation pathway and related enzymes overexpressed in tumor cells are shown in Figure 2.

\section{Conclusion}

Understanding the metabolic alteration and metabolism-related enzyme deregulation in GI malignancies opens up a new insight into the treatment of these cancers. Cancer cells need to have sufficient energy and metabolic intermediates for production of molecules to promote proliferation, migration, and metastasis. Metabolic reprogramming such as the Warburg effect and lipogenesis and their critical enzymes that take place in tumor cells contribute to a condition for tumor survival and growth, so metabolic signaling pathways have become appropriate targets for therapeutic intervention in malignancies. GI tumors are progressive with poor outcomes, and therefore more investigation about the difference between metabolic pathways between GI tumors and normal cells and introduction of the effective agents to inhibit metabolism-related enzyme can reduce tumor growth and improve patient outcomes.

\section{Conflict of Interest Statement}

There are no conflicts of interest in this study.

\section{Funding Sources}

No funding was received for this study.

\section{Author Contributions}

Ahmad Raeisi and Milad Shahini Shams Abadi interpreted and collected data for the work; G.A. Ferns revised the manuscript for intellectual content and commented; MohammadHassan Arjmand designed the work and approved the final version to be published.

\section{References}

1 Boroughs LK, DeBerardinis RJ. Metabolic pathways promoting cancer cell survival and growth. Nat Cell Biol. 2015;17(4):351-9.

2 Dang CV. Links between metabolism and cancer. Genes Dev. 2012;26(9):877-90.

3 Egeblad M, Nakasone ES, Werb Z. Tumors as organs: complex tissues that interface with the entire organism. Dev Cell. 2010;18(6):884901.

$4 \mathrm{Kim}$ J, DeBerardinis RJ. Mechanisms and implications of metabolic heterogeneity in cancer. Cell Metab. 2019;30(3):434-46.

5 Lyssiotis CA, Kimmelman AC. Metabolic interactions in the tumor microenvironment. Trends Cell Biol. 2017;27(11):863-75.

6 Plöckinger U, Rindi G, Arnold R, Eriksson B, Krenning EP, de Herder WW, et al. Guidelines for the diagnosis and treatment of neuroendocrine gastrointestinal tumours. Neuroendocrinology. 2004;80(6):394-424.

7 Hanahan D, Weinberg RA. Hallmarks of cancer: the next generation. cell. 2011;144(5): 646-74.

8 DeBerardinis RJ, Lum JJ, Hatzivassiliou G, Thompson CB. The biology of cancer: metabolic reprogramming fuels cell growth and proliferation. Cell Metab. 2008;7(1):11-20.

9 Romero-Garcia S, Lopez-Gonzalez JS, BáezViveros JL, Aguilar-Cazares D, Prado-Garcia
H. Tumor cell metabolism: an integral view. Cancer Biol Ther. 2011;12:939-48.

10 Warburg O. On the origin of cancer cells. Science. 1956;123(3191):309-14.

11 Warburg O. On respiratory impairment in cancer cells. Science. 1956;124(3215):26970.

12 Gatenby RA, Gillies RJ. Why do cancers have high aerobic glycolysis? Nat Rev Cancer. 2004;4(11):891-9.

$13 \mathrm{Lu} \mathrm{H}$, Forbes RA, Verma A. Hypoxia-inducible factor 1 activation by aerobic glycolysis implicates the Warburg effect in carcinogenesis. J Biol Chem. 2002;277(26):23111-5.

14 He C, Jiang H, Geng S, Sheng H, Shen X, Zhang $\mathrm{X}$, et al. Expression and prognostic value of c-Myc and Fas (CD95/APO1) in patients with pancreatic cancer. Int J Clin Exp Pathol. 2014;7(2):742.

15 de Souza CRT, Leal MF, Calcagno DQ, Costa Sozinho EK, Borges Bdo N, Montenegro RC, et al. MYC deregulation in gastric cancer and its clinicopathological implications. PloS One. 2013;8(5):e64420.

16 Mathupala SP, Ko YH, Pedersen PL. Hexokinase- 2 bound to mitochondria: cancer's stygian link to the "Warburg Effect" and a pivotal target for effective therapy. Semin Cancer Biol. 2009;19:17-24.
17 Wang P, Mai C, Wei YL, Zhao JJ, Hu YM, Zeng ZL, et al. Decreased expression of the mitochondrial metabolic enzyme aconitase (ACO2) is associated with poor prognosis in gastric cancer. Med Oncol. 2013;30(2):552.

18 Kwee SA, Hernandez B, Chan O, Wong L. Choline kinase alpha and hexokinase-2 protein expression in hepatocellular carcinoma: association with survival. PLoS One. 2012; 7(10):e46591.

19 Paudyal B, Paudyal P, Oriuchi N, Tsushima Y, Nakajima T, Endo K. Clinical implication of glucose transport and metabolism evaluated by 18 F-FDG PET in hepatocellular carcinoma. Int J Oncol. 2008;33(5):1047-54.

20 Cao X, Fang L, Gibbs S, Huang Y, Dai Z, Wen $\mathrm{P}$, et al. Glucose uptake inhibitor sensitizes cancer cells to daunorubicin and overcomes drug resistance in hypoxia. Cancer Chemother Pharmacol. 2007;59(4):495-505.

21 Christofk HR, Vander Heiden MG, Harris MH, Ramanathan A, Gerszten RE, Wei R, et al. The M2 splice isoform of pyruvate kinase is important for cancer metabolism and tumour growth. Nature. 2008;452(7184):230-3.

22 Christofk HR, Vander Heiden MG, Wu N, Asara JM, Cantley LC. Pyruvate kinase M2 is a phosphotyrosine-binding protein. Nature. 2008;452(7184):181-6. 
23 Yang W, Xia Y, Hawke D, Li X, Liang J, Xing $\mathrm{D}$, et al. PKM2 phosphorylates histone $\mathrm{H} 3$ and promotes gene transcription and tumorigenesis. Cell. 2012;150(4):685-96.

$24 \mathrm{Wu} \mathrm{S}$, Le H. Dual roles of PKM2 in cancer metabolism. Acta Biochim Biophys Sin. 2013; 45(1):27-35.

25 Zhang X, He C, He C, Chen B, Liu Y, Kong M, et al. Nuclear PKM2 expression predicts poor prognosis in patients with esophageal squamous cell carcinoma. Pathol Res Pract. 2013; 209(8):510-5.

26 Wu J, Hu L, Chen M, Cao W, Chen H, He T. Pyruvate kinase M2 overexpression and poor prognosis in solid tumors of digestive system: evidence from 16 cohort studies. Onco Targets Ther. 2016;9:4277.

27 Li T, Han J, Jia L, Hu X, Chen L, Wang Y. PKM2 coordinates glycolysis with mitochondrial fusion and oxidative phosphorylation. Protein Cell. 2019;10(8):583-94.

28 Tang JC, Zhao J, Long F, Chen JY., Mu B, Jiang $Z$, et al. Efficacy of Shikonin against esophageal cancer cells and its possible mechanisms in vitro and in vivo. J. Cancer. 2018; 9(1):32.

29 Li W, Zhang C, Ren A, Li T, Jin R, Li G, et al. Shikonin suppresses skin carcinogenesis via inhibiting cell proliferation. PLoS One. 2015; 10(5):e0126459.

30 Hur H, Xuan Y, Kim YB, Lee G, Shim W, Yun J, et al. Expression of pyruvate dehydrogenase kinase- 1 in gastric cancer as a potential therapeutic target. Int J Oncol. 2013;42(1):44-54.

31 Lu CW, Lin SC, Chien CW, Lin SC, Lee CT, Lin BW, et al. Overexpression of pyruvate dehydrogenase kinase 3 increases drug resistance and early recurrence in colon cancer. Am J Pathol. 2011;179(3):1405-14.

32 Tong J, Xie G, He J, Li J, Pan F, Liang H. Synergistic antitumor effect of dichloroacetate in combination with 5-fluorouracil in colorectal cancer. J Biomed Biotechnol. 2011;2011: 740564.

33 Shen YC, Ou DL, Hsu C, Lin KL, Chang CY, Lin CY, et al. Activating oxidative phosphorylation by a pyruvate dehydrogenase kinase inhibitor overcomes sorafenib resistance of hepatocellular carcinoma. Br J Cancer. 2013; 108(1):72-81.

34 Moreno-Sánchez R, Marín-Hernández A, Gallardo-Pérez JC, Quezada H, Encalada R, Rodríguez-Enríquez S, et al. Phosphofructokinase type 1 kinetics, isoform expression, and gene polymorphisms in cancer cells. J Cell Biochem. 2012;113:1692-703.

35 Yi W, Clark PM, Mason DE, Keenan MC, Hill C, Goddard WA, et al. Phosphofructokinase 1 glycosylation regulates cell growth and metabolism. Science. 2012;337(6097):975-80.

36 Vora S, Halper JP, Knowles DM. Alterations in the activity and isozymic profile of human phosphofructokinase during malignant transformation in vivo and in vitro: transformation-and progression-linked discriminants of malignancy. Cancer Res. 1985;45: 2993-3001.
37 Niki T, Tsutsui S, Hirose S, Aradono S, Sugimoto Y, Takeshita K, et al. Galectin-9 is a high affinity IgE-binding lectin with anti-allergic effect by blocking IgE-antigen complex formation. J Biol Chem. 2009;284(47): 32344-52.

38 Semenza GL, Roth PH, Fang HM, Wang GL. Transcriptional regulation of genes encoding glycolytic enzymes by hypoxia-inducible factor 1. J Biol Chem. 1994;269(38):2375763.

39 Zhu W, Ye L, Zhang J, Yu P, Wang H, Ye Z, et al. PFK15, a small molecule inhibitor of PFKFB3, induces cell cycle arrest, apoptosis and inhibits invasion in gastric cancer. PLoS One. 2016;11(9):e0163768

40 Korkeila E, Jaakkola PM, Syrjänen K, Pyrhönen S, Sundström J. Pronounced tumour regression after radiotherapy is associated with negative/weak glucose transporter-1 expression in rectal cancer. Anticancer Res. 2011;31(1):311-5.

41 Brophy S, Sheehan KM, McNamara DA, Deasy J, Bouchier-Hayes DJ, Kay EW. GLUT1 expression and response to chemoradiotherapy in rectal cancer. Int J Cancer. 2009; 125(12):2778-82.

42 Kawamura T, Kusakabe T, Sugino T, Watanabe K, Fukuda T, Nashimoto A, et al. Expression of glucose transporter-1 in human gastric carcinoma: association with tumor aggressiveness, metastasis, and patient survival. Cancer. 2001;92:634-41.

43 Sawayama H, Ishimoto T, Watanabe M, Yoshida N, Baba Y, Sugihara H, et al. High expression of glucose transporter 1 on primary lesions of esophageal squamous cell carcinoma is associated with hematogenous recurrence. Ann Surg Oncol. 2014;21(5):1756-62.

44 Kitamura K, Hatano E, Higashi T, Narita M, Seo S, Nakamoto Y, et al. Proliferative activity in hepatocellular carcinoma is closely correlated with glucose metabolism but not angiogenesis. J Hepatol. 2011;55(4):846-57.

45 Dang CV, Le A, Gao P. MYC-induced cancer cell energy metabolism and therapeutic opportunities. Clin Cancer Res. 2009;15(21): 6479-83.

46 Griffiths EA, Pritchard SA, Welch IM, Price $\mathrm{PM}$, West CM. Is the hypoxia-inducible factor pathway important in gastric cancer? Eur J Cancer. 2005;41(18):2792-805.

47 Röhrig F, Schulze A. The multifaceted roles of fatty acid synthesis in cancer. Nat Rev Cancer. 2016;16(11):732.

48 Kuhajda FP. Fatty-acid synthase and human cancer: new perspectives on its role in tumor biology. Nutrition. 2000;16(3):202-8.

49 de Cedrón MG, de Molina AR. Precision nutrition to target lipid metabolism alterations in cancer. Prec Med Invest Pract Prov. 2020: 291-9.

50 Nieman KM, Kenny HA, Penicka CV, Ladanyi A, Buell-Gutbrod R, Zillhardt MR, et al. Adipocytes promote ovarian cancer metastasis and provide energy for rapid tumor growth. Nat Med. 2011;17(11):1498.
51 Yarla NS, Bishayee A, Sethi G, Reddanna P, Kalle AM, Dhananjaya BL, et al. Targeting arachidonic acid pathway by natural products for cancer prevention and therapy. Semin Cancer Biol. 2016.

52 Li S, Qiu L, Wu B, Shen H, Zhu J, Zhou L, et al. TOFA suppresses ovarian cancer cell growth in vitro and in vivo. Mol Med Rep. 2013;8(2):373-8.

53 Moghaddam AA, Woodward M, Huxley R. Obesity and risk of colorectal cancer: a metaanalysis of 31 studies with 70,000 events. Cancer Epidemiol Biomarkers Prev. 2007;16(12): 2533-47.

54 Larsson SC, Wolk A. Overweight, obesity and risk of liver cancer: a meta-analysis of cohort studies. Br J Cancer. 2007;97(7):1005-8.

55 Dobbins M, Decorby K, Choi BCK. The association between obesity and cancer risk: a meta-analysis of observational studies from 1985 to 2011. ISRN Prev Med. 2013;2013:1.

56 Beloribi-Djefaflia S, Vasseur S, Guillaumond F. Lipid metabolic reprogramming in cancer cells. Oncogenesis. 2016;5:e189.

57 Bauer DE, Hatzivassiliou G, Zhao F, Andreadis C, Thompson CB. ATP citrate lyase is an important component of cell growth and transformation. Oncogene. 2005;24(41): 6314-22.

58 Qian X, Hu J, Zhao J, Chen H. ATP citrate lyase expression is associated with advanced stage and prognosis in gastric adenocarcinoma. Int J Clin Exp Med. 2015;8(5):7855

59 Zhou Y, Bollu LR, Tozzi F, Ye X, Bhattacharya $\mathrm{R}$, Gao G, et al. ATP citrate lyase mediates resistance of colorectal cancer cells to SN38. Mol Cancer Ther. 2013;12(12):2782-91.

60 Watson JA, Fang M, Lowenstein JM. Tricarballylate and hydroxycitrate: substrate and inhibitor of ATP: citrate oxaloacetate lyase. Arch Biochem Biophys. 1969;135(1): 209-17.

61 Moffett SA, Bhandari AK, Ravindranath B. Hydroxycitric acid concentrate and food products prepared therefrom. Google Patents. 1997.

62 Wei J, Tong L. Crystal structure of the $500-\mathrm{kDa}$ yeast acetyl-CoA carboxylase holoenzyme dimer. Nature. 2015;526(7575): 723-7.

63 Fang W, Cui H, Yu D, Chen Y, Wang J, Yu G. Increased expression of phospho-acetyl-CoA carboxylase protein is an independent prognostic factor for human gastric cancer without lymph node metastasis. Med Oncol. 2014; 31(7):15.

64 Calvisi DF, Frau M, Tomasi ML, Feo F, Pascale RM. Deregulation of signalling pathways in prognostic subtypes of hepatocellular carcinoma: novel insights from interspecies comparison. Biochim Biophy Acta. 2012; 1826(1):215-37.

65 Nishi K, Suzuki K, Sawamoto J, Tokizawa Y, Iwase Y, Yumita N, et al. Inhibition of fatty acid synthesis induces apoptosis of human pancreatic cancer cells. Anticancer Res. 2016; 36(9):4655-60. 
66 Menendez JA, Vellon L, Mehmi I, Oza BP, Ropero S, Colomer R, et al. Inhibition of fatty acid synthase (FAS) suppresses HER2/neu (erbB-2) oncogene overexpression in cancer cells. Proc Natl Acad Sci U S A. 2004;101(29): 10715-20.

67 Wang H, Xi Q, Wu G. Fatty acid synthase regulates invasion and metastasis of colorectal cancer via Wnt signaling pathway. Cancer Med. 2016;5(7):1599-606.

68 Hiraga T, Ito S, Nakamura H. Cancer stemlike cell marker CD44 promotes bone metastases by enhancing tumorigenicity, cell motility, and hyaluronan production. Cancer Res. 2013;73(13):4112-22

$69 \mathrm{Gu}$ L, Zhu Y, Lin X, Lu B, Zhou X, Zhou F, et al. The IKK $\beta$-USP30-ACLY axis controls lipogenesis and tumorigenesis. Hepatology. 2021;73(1):160-74.

70 Flavin R, Peluso S, Nguyen PL, Loda M. Fatty acid synthase as a potential therapeutic target in cancer. Future Oncol. 2010;6(4):551-62.

71 Pizer ES, Wood FD, Heine HS, Romantsev FE, Pasternack GR, Kuhajda FP. Inhibition of fatty acid synthesis delays disease progression in a xenograft model of ovarian cancer. Cancer Res. 1996;56(6):1189-93.

72 Murata S, Yanagisawa K, Fukunaga K, Oda T, Kobayashi A, Sasaki R, et al. Fatty acid synthase inhibitor cerulenin suppresses liver metastasis of colon cancer in mice. Cancer Sci. 2010;101(8):1861-5.

73 Shiragami R, Murata S, Kosugi C, Tezuka T, Yamazaki M, Hirano A, et al. Enhanced antitumor activity of cerulenin combined with oxaliplatin in human colon cancer cells. Int J Oncol. 2013;43(2):431-8.

74 Duan J, Chen L, Zhou M, Zhang J, Sun L, Huang N, et al. MACC1 decreases the chemosensitivity of gastric cancer cells to oxaliplatin by regulating FASN expression. Oncol Rep. 2017;37(5):2583-92.

75 Ma S, Sun W, Gao L, Liu S. Therapeutic targets of hypercholesterolemia: HMGCR and LDLR. Dmso. 2019;12:1543-53.

76 Gray RT, Loughrey MB, Bankhead P, Cardwell CR, McQuaid S, O'Neill RF, et al. Statin use, candidate mevalonate pathway biomarkers, and colon cancer survival in a population-based cohort study. Br J Cancer. 2017;116(12):1652-9.

77 Che L, Chi W, Qiao Y, Zhang J, Song X, Liu $\mathrm{Y}$, et al. Cholesterol biosynthesis supports the growth of hepatocarcinoma lesions depleted of fatty acid synthase in mice and humans. Gut. 2020;69(1):177-86.

78 Shi J, Zhu J, Zhao H, Zhong C, Xu Z, Yao F. Mevalonate pathway is a therapeutic target in esophageal squamous cell carcinoma. Tumour Biol. 2013;34(1):429-35.
79 Zhong C, Fan L, Yao F, Shi J, Fang W, Zhao H. HMGCR is necessary for the tumorigenecity of esophageal squamous cell carcinoma and is regulated by Myc. Tumour Biol. 2014;35(5):41239.

80 Garber K. Energy boost: the Warburg effect returns in a new theory of cancer. J Natl Cancer Inst. 2004;96(24):1805-6.

81 Price DT, Coleman RE, Liao RP, Robertson CN, Polascik TJ, DeGrado TR. Comparison of [18 F]fluorocholine and [18 F]fluorodeoxyglucose for positron emission tomography of androgen dependent and androgen independent prostate cancer. J Urol. 2002;168(1):273-80.

82 Effert PJ, Bares R, Handt S, Wolff JM, Büll U, Jakse G. Metabolic imaging of untreated prostate cancer by positron emission tomography with 18fluorine-labeled deoxyglucose. J Urol. 1996;155(3):994-8.

83 Pike LS, Smift AL, Croteau NJ, Ferrick DA, Wu M. Inhibition of fatty acid oxidation by etomoxir impairs NADPH production and increases reactive oxygen species resulting in ATP depletion and cell death in human glioblastoma cells. Biochim Biophys Acta. 2011;1807(6):726-34.

84 Toshima T, Shirabe K, Matsumoto Y, Yoshiya S, Ikegami T, Yoshizumi T, et al. Autophagy enhances hepatocellular carcinoma progression by activation of mitochondrial $\beta$-oxidation. J Gastroenterol. 2014;49(5):907-16. 